\title{
Prevalence of Antimicrobial Resistance of Common Bacterial Isolates Before and During COVID-19 Pandemic in Armed Forces Hospital Jazan, Saudi Arabia
}

Manal AlDiba, Ahmad Mohammad Hassan Daghriri, Essa Mohammed Essa Jamali, Ahmed Alzahrani, Ahmed Bijad Alsharif, Haider Ghalip Almudeer, Hassan Mosa Ahmed Khobrani, Nabil

Turki Madhi Hakami, Wagih Mommtaz Ghannam, and Hassan Mohamed Alasy

\section{ABSTRACT}

Background: Antimicrobial resistance (AMR) is one of the top global public health issues. After the pandemic of corona virus 2019 (COVID-19), AMR became a challenging complex that needs to be addressed during the corona pandemic.

Objectives: Evaluating the prevalence of antimicrobial resistance (AMR) before and during COVID-19 pandemic.

Methods: This is a retrospective cross-sectional study that was conducted at Armed Forces Hospital Jazan, Saudi Arabia using positive cultures`results from microbiology department.

Results: Escherichia coli, Pseudomonas aeruginosa, Staphylococcus aureus and Klebsiella pneumoniae were the most prevalent microorganisms isolated from the patients. E. coli was the most isolated organism from patients with infections such as: COVID-19 fever, sepsis, urinary tract infections and wound infection. There was no significant change between 2019 and 2020 regarding AMR prevalence.

Conclusion: There was no significant difference in the AMR before and during the COVID-19 era thus other large population studies must be conducted to evaluate worldwide evolution of AMR during COVID-19 pandemic.

Keywords: Antibiotics, antimicrobial resistance, coronavirus critical illness, COVID-19 pandemic, drug resistance, KSA, sepsis, urinary tract infection.

Submitted: September 5, 2021

Published: September 29, 2021

ISSN: 2593-8339

DOI: $10.24018 /$ ejmed.2021.3.5.1047

\section{AlDiba}

Microbiology Department, Armed Forces Hospital Jazan, Saudi Arabia.

(e-mail: manalaldiba@yahoo.com) A. M. H. Daghriri

Microbiology Department, Armed Forces Hospital Jazan, Saudi Arabia.

(e-mail: a.vip.1402@gmail.com) E. M. E. Jamali

Microbiology Department, Armed Forces Hospital Jazan, Saudi Arabia.

(e-mail: saudi_code@hotmail.com)

A. Alzahrani

Preventive Medicine Department, Armed Forced Hospital, Saudi Arabia.

(e-mail: ahmedsaudsaad@gmail.com)

A. B. Alsharif

Armed Forces Hospital Jazan, Saudi Arabia.

(e-mail: dr.a.b.r3@hotmail.com)

H. G. Almudeer

Microbiology Department, Armed

Forces Hospital Jazan, Saudi Arabia.

(e-mail: hyder1818@gmail.com)

H. M. A. Khobrani

Microbiology Department, Armed

Forces Hospital Jazan, Saudi Arabia.

(e-mail: mrr.ok@hotmail.com)

N. T. K. M. Hakami

Microbiology Department, Armed

Forces Hospital Jazan, Saudi Arabia.

(e-mail: nabeelt33@gmail.com)

W. M. Ghannam*

Mansoura Faculty of Medicine,

Mansoua University, Egypt.

(e-mail:wghnnam@gmail.com)

H. M. Alasy

Tanta Faculty of Medicine, Tanta

University, Egypt.

(e-mail: drhassanalasy @yahoo.com)

*Corresponding Author 


\section{BACKGROUND}

The improper and increased usage of antibiotics have led to development and spreading of antimicrobial resistance (AMR), which is considered a major worldwide health problem. According to the antimicrobial stewardship programs, adjusting the proper usage of antibiotics with correct dose and duration could outcome or at least limit the AMR side effects [1].

Recently, studies showed a significant increase in AMR resulting from COVID-19 pandemic [2]-[4]. Although the world is suffering to control COVID-19 pandemic, the AMR outbreaks development should be considered. Also, multidrug resistant organisms (MDROs) were noticed during that pandemic as carbapenem resistant Enterobacteriaceae (CRE) and methicillin resistant Staphylococcus aureus (MRSA) Frontiers in microbiology. Several studies in Europe showed an increase in MDROs infections during COVID-19 pandemic but other studies in France and Spain did not show increase in MDROs infections. Therefore, the impact of COVID-19 pandemic on AMR will only become clear in the coming years as a lot of published data would become available [5].

Bacterial co-infection during viral infections are significant cause of morbidity and mortality but the clinical evidence suggests that bacterial co-infections rates for the COVID-19 patients are very low but antibiotic prescribing remain high [4]. It was noted that about $72 \%$ of COVID-19 patients were treated with antibiotic even when not clinically indicated and this heavy use of empiric antibiotics led to high rate of AMR [6].

The hypothesis of this study suggests that the more prevalence of COVID-19 patients', the more inappropriate use of antibiotics could occur which may lead to significant increase in AMR. Most of the published data were from Europe and Western countries but to our knowledge no similar studies have been conducted at local or national level in KSA till to date, therefore, our study is important to detect the prevalence of AMR before and during COVID-19 pandemic at local level in Jazan, Saudi Arabia.

\section{METHODS}

\section{A. Study Design and Area}

Two years retrospective cross sectional study was conducted at Armed Forces Hospital Jazan (AFHJ), Kingdom of Saudi Arabia (KSA). The hospital is about 70 bedded hospitals, and it is Central Board for Accreditation of Healthcare Institutions (CBAHI) accredited hospital.

\section{B. Study Population}

All patients (inpatients and outpatients) who were suspected to have bacterial infections and samples were collected from them and cultured for possible pathogen. Full two years data of all positive culture results between 1 January 2019 and 31 December 2020 were obtained from AFHJ microbiology laboratory including full bacterial identification and susceptibility tests results. The inclusion criteria were all positive cases with complete data and Clinical and Laboratory Standards Institutes (CLSI) states that an organism of more than or equal to 30 is optimal for annual antibiogram inclusion [7].

\section{Sample Collection}

Positive bacterial cultures were collected from different types of samples such as urine, respiratory sources, wounds swab, and blood. All samples collected from inpatients and outpatients who visited AFHJ in Jazan using standard laboratory procedures between January 2019 and December 2020 [7].

\section{Study Tools}

\section{1) Identification of Bacterial Etiology}

All the specimens were processed according to the CLSI guidelines [8]. Samples were inoculated on different types of agar plates (Saudi Prepared Media Laboratory Company, Riyadh) according to sample type using sterile disposable loops. Plates were incubated at $35-37^{\circ} \mathrm{C}$ for $24-48$ hours.

Primary identification of growth was done by the manual methods such as colony morphology, Gram staining and culture characteristics on the plates. Further identification of bacterial species was done using BD phoenix M50 automated system using appropriate panels for the Gram-negative bacteria and for the Gram-positive bacteria according to the manufacturer instructions.

\section{2) Antimicrobial Susceptibility Testing}

Identified bacteria were screened for their antimicrobial susceptibility patterns using BD phoenix M50 automated system according to the manufacturer's instructions then for the confirmation, the epsilometer (E-test) was conducted.

\section{3) Antimicrobials Resistance Patterns}

The data of antimicrobials resistance patterns are presented in terms of total numbers of isolate and their percentage. Multidrug resistance was defined as the resistance of isolate to two or more antimicrobials.

\section{4) Internal Quality Control Assurance}

E. coli American Type Culture Collection (ATCC 25922), E. faecalis (ATCC 51299), S. aureus (ATCC 29213), and $P$. aeruginosa (ATCC 27853) were used as quality control strains.

\section{5) Data Collection}

Electronically completed results from the microbiology laboratory computer system in AFHJ in Jazan. Those positive results had been obtained using some tools such as agar plate, Gram staining, and automated machine for bacterial identification and antimicrobial susceptibility testing (AST).

\section{E. Ethical Considerations}

Ethical approval was obtained from Evidence Based and Research Ethics Committee at AFHJ. The collected data will be safe and confidential for research purposes.

\section{F. Statistical Analysis}

The data were fed up into windows and analyzed using SPSS V.22. The data were shown as distributions and percentages. Chi square test was used to calculate p-values. 


\section{RESULTS}

\section{A. Demographics of the Included Subjects}

Table I showed the demographics of the included subjects n=249 and 264 in 2019 and 2020 respectively including age, gender, and hospital units. Most of the subjects were males
$61.4 \%$ and $71.2 \%$ during 2019 and 2020, respectively. The age of included subjects ranged from younger than 12 years old to 80 years old. During 2019,26.5\% of the patients were admitted to intensive care units (ICU) unit followed by male ward (MW) and female ward (FW) unit while in 2020 the highest percentage was for MW followed by ICU.

\begin{tabular}{|c|c|c|c|c|c|c|c|c|c|}
\hline & & \multicolumn{2}{|c|}{2019} & \multicolumn{2}{|c|}{2020} & \multicolumn{2}{|c|}{ Total } & \multicolumn{2}{|c|}{ Chi-square } \\
\hline & & $\mathrm{N}$ & $\%$ & $\mathrm{~N}$ & $\%$ & $\mathrm{~N}$ & $\%$ & $\mathrm{X}^{2}$ & P-value \\
\hline \multirow{7}{*}{ Age } & $<12$ & 29 & $11.6 \%$ & 14 & $5.3 \%$ & 43 & $8.4 \%$ & \multirow{7}{*}{13.263} & \multirow{7}{*}{0.039} \\
\hline & $12-35$ & 23 & $9.2 \%$ & 40 & $15.2 \%$ & 63 & $12.3 \%$ & & \\
\hline & $35-50$ & 46 & $18.5 \%$ & 44 & $16.7 \%$ & 90 & $17.5 \%$ & & \\
\hline & $50-60$ & 37 & $14.9 \%$ & 39 & $14.8 \%$ & 76 & $14.8 \%$ & & \\
\hline & $60-70$ & 48 & $19.3 \%$ & 67 & $25.4 \%$ & 115 & $22.4 \%$ & & \\
\hline & $70-80$ & 42 & $16.9 \%$ & 36 & $13.6 \%$ & 78 & $15.2 \%$ & & \\
\hline & $>80$ & 24 & $9.6 \%$ & 24 & $9.1 \%$ & 48 & $9.4 \%$ & & \\
\hline \multirow{2}{*}{ Sex } & Female & 96 & $38.6 \%$ & 76 & $28.8 \%$ & 172 & $33.5 \%$ & \multirow{2}{*}{5.491} & \multirow{2}{*}{0.019} \\
\hline & Male & 153 & $61.4 \%$ & 188 & $71.2 \%$ & 341 & $66.5 \%$ & & \\
\hline \multirow{6}{*}{ UNITS } & fw & 52 & $20.9 \%$ & 41 & $15.5 \%$ & 93 & $18.1 \%$ & \multirow{6}{*}{29.760} & \multirow{6}{*}{0.000} \\
\hline & $\mathrm{icu}$ & 66 & $26.5 \%$ & 63 & $23.9 \%$ & 129 & $25.1 \%$ & & \\
\hline & msw & 36 & $14.5 \%$ & 43 & $16.3 \%$ & 79 & $15.4 \%$ & & \\
\hline & $\mathrm{mw}$ & 56 & $22.5 \%$ & 67 & $25.4 \%$ & 123 & $24.0 \%$ & & \\
\hline & Out Patient & 9 & $3.6 \%$ & 38 & $14.4 \%$ & 47 & $9.2 \%$ & & \\
\hline & Pedia & 30 & $12.0 \%$ & 12 & $4.5 \%$ & 42 & $8.2 \%$ & & \\
\hline
\end{tabular}

\section{B. Distribution of the Studied Samples regarding to Sample Type, Isolated Organisms and Diagnosis}

TABLE II: DiSTRIBUTION OF THE STUDIED SAMPLES REGARDING SAMPLE TYPE, ISOLATED ORGANS AND DIAGNOSIS IN 2019 AND 2020

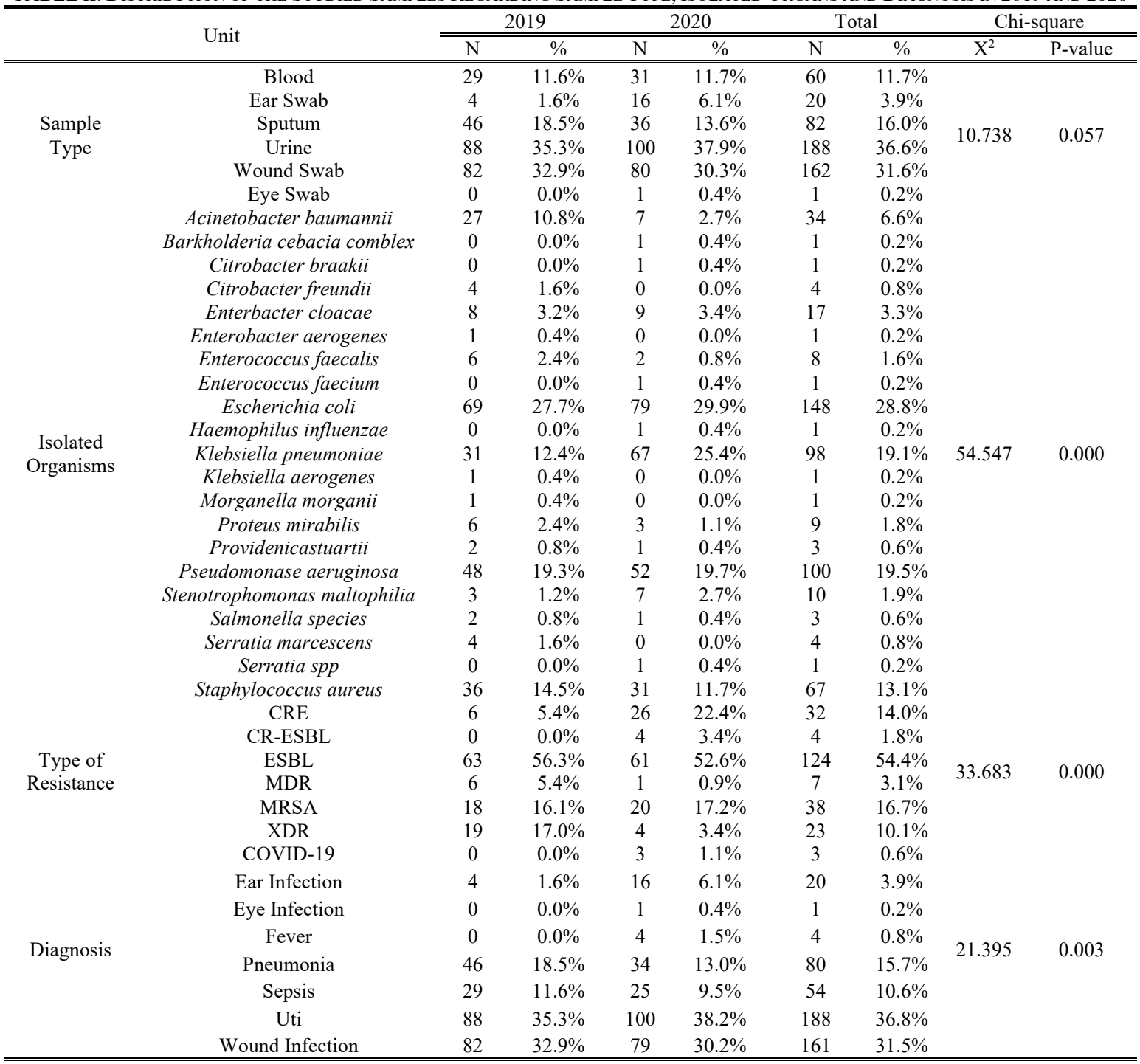


Table II showed the distribution of the studied samples regarding to sample type, isolated organisms, and diagnosis as the majority of the samples were taken from urine, wound swabs followed by sputum and blood. The most isolated organisms in 2019 were Escherichia coli (27.7\%), Pseudomonas aeruginosa (19.3\%), and Staphylococcus aureus (14.5\%) while in 2020 the isolated organisms were Escherichia coli (29.9\%), Klebsiella pneumoniae (25.4\%), Pseudomonas aeruginosa (19.7\%), and Staphylococcus aureus (11.7\%). Extended Spectrum Beta-Lactamase (ESBL) was the most frequent resistance type in 2019 $(56.3 \%)$ and in $2020(52.6 \%)$. It was followed by Extensive drug resistance (XDR) (17\%) and methicillin-resistant Staphylococcus aureus (MRSA) (16.1\%) in 2019 and Carbapenem-resistant Enterobacteriaceae (CRE) (22.4\%) and MRSA (17.2\%) in 2020. The diagnosis of the type of infection showed that urinary tract infection (UTI), wound infection, pneumonia and sepsis were prevalent during 2019 and 2020. On the other hand, COVID-19 and eye infection were the least frequent infections either in 2019 or 2020.

\section{Antibiotic Sensitivity Test}

The microorganisms were very sensitive to Amikacin,
Daptomycin, Ertapenem, Erythromycin, clindamycin, Imipenem, Linezolid, Meropenem, Moxifloxacin, Mupirocin, Rifampin, Teicoplanin and Vancomycin. There was a moderate sensitivity to Ciprofloxacin, Nitrofurantoin, Piperacillin-tazobactam, Tigecycline, and Trimethoprim/sulphamethoxazole. They showed a high resistance pattern to Amoxicillin-clavulanic, Ampicillin, Cefotaxime, Cefoxitin, Ceftazidime, Ceftriaxone, Cefuroxime, Oxacillin, and Penicillin-g (Table III and Fig. 1).

Table IV and Fig. 2 showed that the antibiotics' resistance and sensitivity pattern showed no significant change between 2019 and 2020 except for Amoxicillin-clavulanic which showed significant decrease in the resistance pattern to such antibiotic.

\section{Association between the Isolated Organisms and Resistance Pattern}

Antimicrobial susceptibility patterns of bacterial strains isolated in association with antibiotic resistance is presented in Table V.

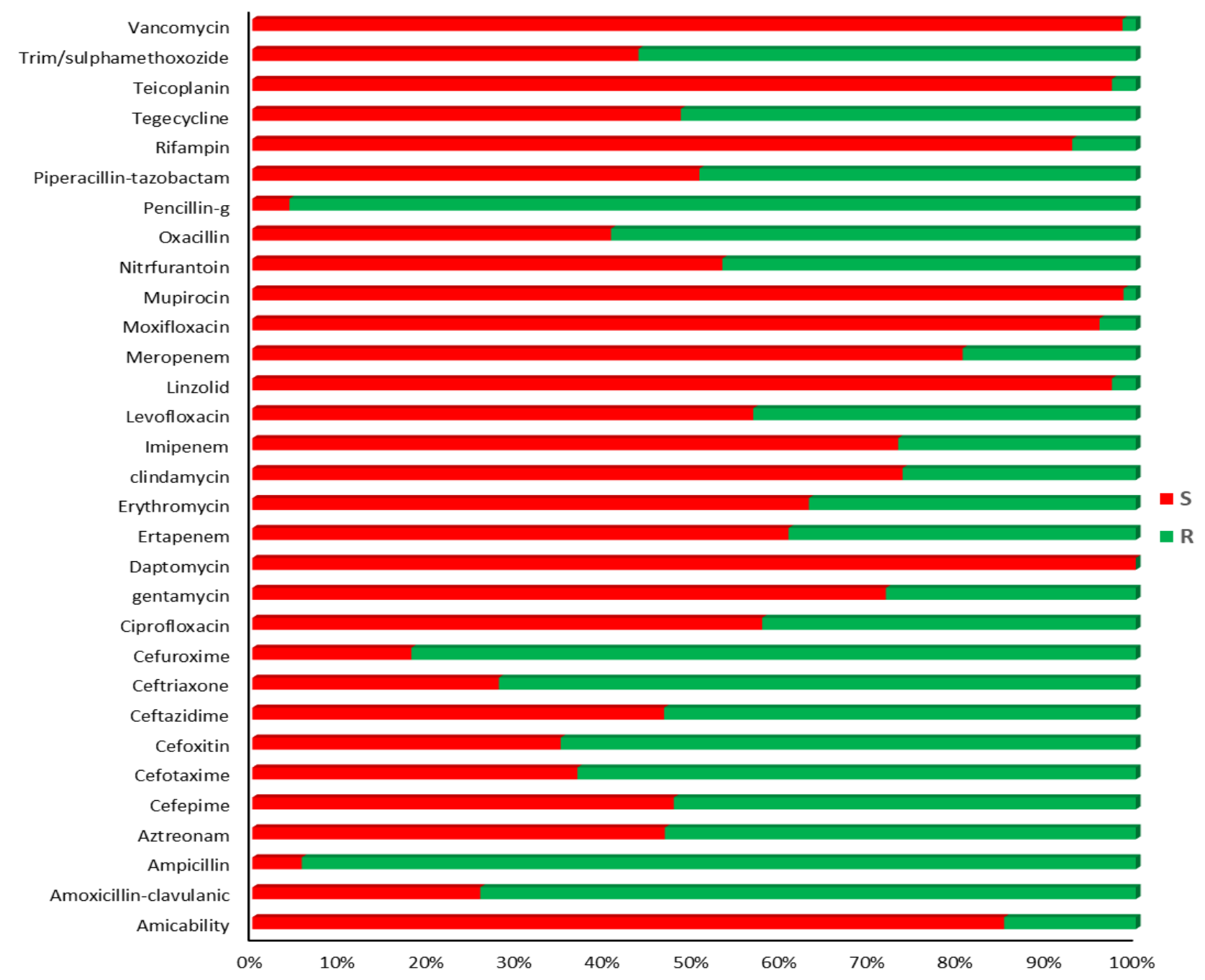

Fig. 1. Antibiotic sensitivity test. 
TABLE III: ANTIBIOTIC SENSITIVITY TEST

\begin{tabular}{|c|c|c|c|c|c|c|}
\hline & \multicolumn{2}{|c|}{$\mathrm{S}$} & \multicolumn{2}{|c|}{$\mathrm{R}$} & \multicolumn{2}{|c|}{ Total } \\
\hline & $\mathrm{N}$ & $\%$ & $\mathrm{~N}$ & $\%$ & $\mathrm{~N}$ & $\%$ \\
\hline Amikacin & 371 & $85.1 \%$ & 65 & $14.9 \%$ & 436 & $100.0 \%$ \\
\hline Amoxicillin-clavulanic & 118 & $25.8 \%$ & 339 & $74.2 \%$ & 457 & $100.0 \%$ \\
\hline Ampicillin & 28 & $5.6 \%$ & 476 & $94.4 \%$ & 504 & $100.0 \%$ \\
\hline Aztreonam & 42 & $46.7 \%$ & 48 & $53.3 \%$ & 90 & $100.0 \%$ \\
\hline Cefepime & 207 & $47.7 \%$ & 227 & $52.3 \%$ & 434 & $100.0 \%$ \\
\hline Cefotaxime & 28 & $36.8 \%$ & 48 & $63.2 \%$ & 76 & $100.0 \%$ \\
\hline Cefoxitin & 44 & $34.9 \%$ & 82 & $65.1 \%$ & 126 & $100.0 \%$ \\
\hline Ceftazidime & 203 & $46.6 \%$ & 233 & $53.4 \%$ & 436 & $100.0 \%$ \\
\hline Ceftriaxone & 122 & $27.9 \%$ & 315 & $72.1 \%$ & 437 & $100.0 \%$ \\
\hline Cefuroxime & 90 & $18.0 \%$ & 411 & $82.0 \%$ & 501 & $100.0 \%$ \\
\hline Ciprofloxacin & 295 & $57.7 \%$ & 216 & $42.3 \%$ & 511 & $100.0 \%$ \\
\hline gentamycin & 365 & $71.7 \%$ & 144 & $28.3 \%$ & 509 & $100.0 \%$ \\
\hline Daptomycin & 72 & $100.0 \%$ & 0 & $0.0 \%$ & 72 & $100.0 \%$ \\
\hline Ertapenem & 263 & $60.7 \%$ & 170 & $39.3 \%$ & 433 & $100.0 \%$ \\
\hline Erythromycin & 46 & $63.0 \%$ & 27 & $37.0 \%$ & 73 & $100.0 \%$ \\
\hline clindamycin & 53 & $73.6 \%$ & 19 & $26.4 \%$ & 72 & $100.0 \%$ \\
\hline Imipenem & 366 & $73.1 \%$ & 135 & $26.9 \%$ & 501 & $100.0 \%$ \\
\hline Levofloxacin & 246 & $56.7 \%$ & 188 & $43.3 \%$ & 434 & $100.0 \%$ \\
\hline Linzolid & 72 & $97.3 \%$ & 2 & $2.7 \%$ & 74 & $100.0 \%$ \\
\hline Meropenem & 349 & $80.4 \%$ & 85 & $19.6 \%$ & 434 & $100.0 \%$ \\
\hline Moxifloxacin & 70 & $95.9 \%$ & 3 & $4.1 \%$ & 73 & $100.0 \%$ \\
\hline Mupirocin & 70 & $98.6 \%$ & 1 & $1.4 \%$ & 71 & $100.0 \%$ \\
\hline Nitrfurantoin & 269 & $53.2 \%$ & 237 & $46.8 \%$ & 506 & $100.0 \%$ \\
\hline Oxacillin & 28 & $40.6 \%$ & 41 & $59.4 \%$ & 69 & $100.0 \%$ \\
\hline Pencillin-g & 3 & $4.2 \%$ & 68 & $95.8 \%$ & 71 & $100.0 \%$ \\
\hline Piperacillin-tazobactam & 221 & $50.6 \%$ & 216 & $49.4 \%$ & 437 & $100.0 \%$ \\
\hline Rifampin & 64 & $92.8 \%$ & 5 & $7.2 \%$ & 69 & $100.0 \%$ \\
\hline Tegecycline & 210 & $48.5 \%$ & 223 & $51.5 \%$ & 433 & $100.0 \%$ \\
\hline Teicoplanin & 71 & $97.3 \%$ & 2 & $2.7 \%$ & 73 & $100.0 \%$ \\
\hline $\begin{array}{c}\text { Trimethoprim/ } \\
\text { sulphamethoxazole }\end{array}$ & 220 & $43.7 \%$ & 283 & $56.3 \%$ & 503 & $100.0 \%$ \\
\hline Vancomycin & 66 & $98.5 \%$ & 1 & $1.5 \%$ & 67 & $100.0 \%$ \\
\hline Total & 4672 & $52.0 \%$ & 4310 & $48.0 \%$ & 8982 & $100.0 \%$ \\
\hline
\end{tabular}

TABLE IV: ANTIBIOTIC SENSITIVITY TEST DIFFERENCE BETWEEN 2019 AND 2020

\begin{tabular}{|c|c|c|c|c|c|c|c|c|c|c|c|c|}
\hline & \multicolumn{5}{|c|}{2019} & \multicolumn{5}{|c|}{2020} & \multirow{2}{*}{\multicolumn{2}{|c|}{ Chi-square }} \\
\hline & \multicolumn{2}{|c|}{$\mathrm{S}$} & \multicolumn{2}{|c|}{$\mathrm{R}$} & \multirow{2}{*}{ Total } & \multicolumn{2}{|c|}{$\mathrm{S}$} & \multicolumn{2}{|c|}{$\mathrm{R}$} & \multirow{2}{*}{ Total } & & \\
\hline & $\mathrm{N}$ & $\%$ & $\mathrm{~N}$ & $\%$ & & $\mathrm{~N}$ & $\%$ & $\mathrm{~N}$ & $\%$ & & $\mathrm{X}^{2}$ & P-value \\
\hline Amikacin & 177 & $85.5 \%$ & 30 & $14.5 \%$ & 207 & 194 & $84.7 \%$ & 35 & $15.3 \%$ & 229 & 0.054 & 0.817 \\
\hline Amoxicillin-clavulanic & 40 & $19.3 \%$ & 167 & $80.7 \%$ & 207 & 78 & $31.2 \%$ & 172 & $68.8 \%$ & 250 & 8.480 & $0.004 *$ \\
\hline Ampicillin & 18 & $7.2 \%$ & 231 & $92.8 \%$ & 249 & 10 & $3.9 \%$ & 245 & $96.1 \%$ & 255 & 2.658 & 0.103 \\
\hline Aztreonam & - & - & - & - & - & 42 & $46.7 \%$ & 48 & $53.3 \%$ & 90 & - & - \\
\hline Cefepime & 95 & $45.9 \%$ & 112 & $54.1 \%$ & 207 & 112 & $49.3 \%$ & 115 & $50.7 \%$ & 227 & 0.515 & 0.473 \\
\hline Cefotaxime & 17 & $40.5 \%$ & 25 & $59.5 \%$ & 42 & 11 & $32.4 \%$ & 23 & $67.6 \%$ & 34 & 0.535 & 0.464 \\
\hline Cefoxitin & 17 & $43.6 \%$ & 22 & $56.4 \%$ & 39 & 27 & $31.0 \%$ & 60 & $69.0 \%$ & 87 & 1.838 & 0.175 \\
\hline Ceftazidime & 94 & $45.6 \%$ & 112 & $54.4 \%$ & 206 & 109 & $47.4 \%$ & 121 & $52.6 \%$ & 230 & 0.135 & 0.713 \\
\hline Ceftriaxone & 56 & $27.1 \%$ & 151 & $72.9 \%$ & 207 & 66 & $28.7 \%$ & 164 & $71.3 \%$ & 230 & 0.146 & 0.702 \\
\hline Cefuroxime & 40 & $16.2 \%$ & 207 & $83.8 \%$ & 247 & 50 & $19.7 \%$ & 204 & $80.3 \%$ & 254 & 1.037 & 0.308 \\
\hline Ciprofloxacin & 150 & $60.5 \%$ & 98 & $39.5 \%$ & 248 & 145 & $55.1 \%$ & 118 & $44.9 \%$ & 263 & 1.499 & 0.221 \\
\hline gentamycin & 186 & $75.0 \%$ & 62 & $25.0 \%$ & 248 & 179 & $68.6 \%$ & 82 & $31.4 \%$ & 261 & 2.589 & 0.108 \\
\hline Daptomycin & 41 & $100.0 \%$ & - & - & 41 & 31 & $100.0 \%$ & - & - & 31 & - & - \\
\hline Ertapenem & 118 & $57.6 \%$ & 87 & $42.4 \%$ & 205 & 145 & $63.6 \%$ & 83 & $36.4 \%$ & 228 & 1.649 & 0.199 \\
\hline Erythromycin & 28 & $68.3 \%$ & 13 & $31.7 \%$ & 41 & 18 & $56.3 \%$ & 14 & $43.8 \%$ & 32 & 1.116 & 0.291 \\
\hline clindamycin & 32 & $80.0 \%$ & 8 & $20.0 \%$ & 40 & 21 & $65.6 \%$ & 11 & $34.4 \%$ & 32 & 1.885 & 0.170 \\
\hline Imipenem & 178 & $73.6 \%$ & 64 & $26.4 \%$ & 242 & 188 & $72.6 \%$ & 71 & $27.4 \%$ & 259 & 0.059 & 0.807 \\
\hline Levofloxacin & 123 & $59.4 \%$ & 84 & $40.6 \%$ & 207 & 123 & $54.2 \%$ & 104 & $45.8 \%$ & 227 & 1.210 & 0.271 \\
\hline Linzolid & 41 & $97.6 \%$ & 1 & $2.4 \%$ & 42 & 31 & $96.9 \%$ & 1 & $3.1 \%$ & 32 & 0.038 & 0.846 \\
\hline Meropenem & 167 & $81.1 \%$ & 39 & $18.9 \%$ & 206 & 182 & $79.8 \%$ & 46 & $20.2 \%$ & 228 & 0.106 & 0.744 \\
\hline Moxifloxacin & 38 & $92.7 \%$ & 3 & $7.3 \%$ & 41 & 32 & $100.0 \%$ & 0 & $0.0 \%$ & 32 & 3.561 & 0.059 \\
\hline Mupirocin & 38 & $97.4 \%$ & 1 & $2.6 \%$ & 39 & 32 & $100.0 \%$ & 0 & $0.0 \%$ & 32 & 1.210 & 0.271 \\
\hline Nitrfurantoin & 134 & $53.8 \%$ & 115 & $46.2 \%$ & 249 & 135 & $52.5 \%$ & 122 & $47.5 \%$ & 257 & 0.084 & 0.772 \\
\hline Oxacillin & 17 & $45.9 \%$ & 20 & $54.1 \%$ & 37 & 11 & $34.4 \%$ & 21 & $65.6 \%$ & 32 & 0.957 & 0.328 \\
\hline Pencillin-g & 1 & $2.6 \%$ & 37 & $97.4 \%$ & 38 & 2 & $6.1 \%$ & 31 & $93.9 \%$ & 33 & 0.517 & 0.472 \\
\hline $\begin{array}{l}\text { Piperacillin- } \\
\text { tazobactam }\end{array}$ & 104 & $50.2 \%$ & 103 & $49.8 \%$ & 207 & 117 & $50.9 \%$ & 113 & $49.1 \%$ & 230 & 0.017 & 0.896 \\
\hline Rifampin & 36 & $97.3 \%$ & 1 & $2.7 \%$ & 37 & 28 & $87.5 \%$ & 4 & $12.5 \%$ & 32 & 2.567 & 0.109 \\
\hline Tegecycline & 111 & $53.9 \%$ & 95 & $46.1 \%$ & 206 & 99 & $43.6 \%$ & 128 & $56.4 \%$ & 227 & 4.569 & 0.033 \\
\hline Teicoplanin & 40 & $95.2 \%$ & 2 & $4.8 \%$ & 42 & 31 & $100.0 \%$ & 0 & $0.0 \%$ & 31 & 2.253 & 0.133 \\
\hline Trime/sulpham-ethoxazole & 114 & $45.8 \%$ & 135 & $54.2 \%$ & 249 & 106 & $41.7 \%$ & 148 & $58.3 \%$ & 254 & 0.839 & 0.360 \\
\hline Vancomycin & 37 & $97.4 \%$ & 1 & $2.6 \%$ & 38 & 29 & $100.0 \%$ & 0 & $0.0 \%$ & 29 & 1.146 & 0.284 \\
\hline Total & 2288 & $53.0 \%$ & 2026 & $47.0 \%$ & 4314 & 2384 & $51.1 \%$ & 2284 & $48.9 \%$ & 4668 & & \\
\hline
\end{tabular}


European Journal of Medical and Health Sciences www.ejmed.org

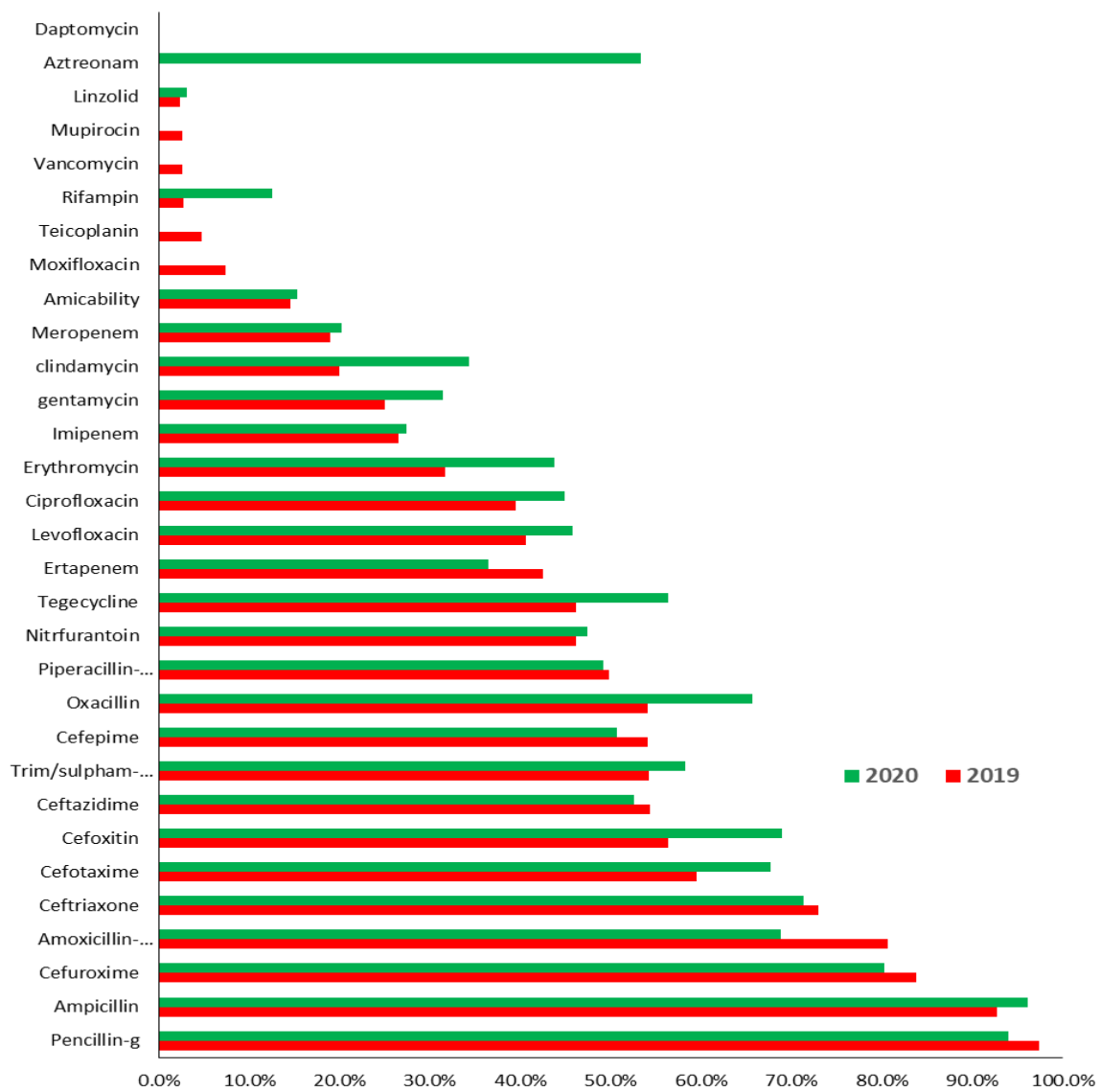

Fig. 2. The antibiotic sensitivity difference between 2019 and 2020.

TABLE V: ASSOCIATION BETWEEN THE ISOLATED ORGANISMS AND RESISTANCE PATTERN (R)

\begin{tabular}{ccccccccc}
\hline \hline & \multicolumn{7}{c}{ Isolated Organisms } \\
\cline { 2 - 9 } $\mathrm{R}$ & \multicolumn{2}{c}{ Escherichia coli } & $\begin{array}{c}\text { Klebsiella } \\
\text { pneumoniae }\end{array}$ & $\begin{array}{c}\text { Pseudomonas } \\
\text { aeruginosa }\end{array}$ & $\begin{array}{c}\text { Staphylococcus } \\
\text { aureus }\end{array}$ \\
\cline { 2 - 9 } & $\mathrm{N}$ & $\%$ & $\mathrm{~N}$ & $\%$ & $\mathrm{~N}$ & $\%$ & $\mathrm{~N}$ & $\%$ \\
\hline \hline Amikacin & 0 & $0.0 \%$ & 24 & $24.5 \%$ & 5 & $5.0 \%$ & - & - \\
Amoxicillin-clavulanic & 90 & $60.8 \%$ & 56 & $57.1 \%$ & 100 & $100.0 \%$ & 15 & $71.4 \%$ \\
Ampicillin & 135 & $91.2 \%$ & 97 & $99.0 \%$ & 100 & $100.0 \%$ & 57 & $96.6 \%$ \\
Aztreonam & 11 & $52.4 \%$ & 13 & $41.9 \%$ & 18 & $69.2 \%$ & - & - \\
Cefepime & 97 & $65.5 \%$ & 51 & $52.0 \%$ & 28 & $28.3 \%$ & - & - \\
Cefotaxime & - & - & - & - & - & - & 39 & $58.2 \%$ \\
Cefoxitin & 2 & $18.2 \%$ & 11 & $61.1 \%$ & 17 & $100.0 \%$ & 39 & $60.0 \%$ \\
Ceftazidime & 102 & $68.9 \%$ & 56 & $57.1 \%$ & 26 & $26.0 \%$ & - & - \\
Ceftriaxone & 102 & $68.9 \%$ & 55 & $56.1 \%$ & 99 & $99.0 \%$ & - & - \\
Cefuroxime & 114 & $77.0 \%$ & 76 & $77.6 \%$ & 99 & $99.0 \%$ & 35 & $58.3 \%$ \\
Ciprofloxacin & 82 & $55.4 \%$ & 40 & $40.8 \%$ & 30 & $30.0 \%$ & 10 & $14.9 \%$ \\
gentamycin & 41 & $27.9 \%$ & 27 & $27.6 \%$ & 15 & $15.0 \%$ & 4 & $6.1 \%$ \\
Daptomycin & - & - & - & - & - & - & 0 & $0.0 \%$ \\
Ertapenem & 7 & $4.7 \%$ & 27 & $27.8 \%$ & 85 & $87.6 \%$ & - & - \\
Erythromycin & - & - & - & - & - & - & 19 & $29.2 \%$ \\
clindamycin & - & - & - & - & - & - & 12 & $18.5 \%$ \\
Imipenem & 6 & $4.1 \%$ & 28 & $28.6 \%$ & 22 & $22.0 \%$ & 39 & $60.0 \%$ \\
Levofloxacin & 80 & $54.1 \%$ & 39 & $40.2 \%$ & 32 & $32.0 \%$ & - & - \\
Linzolid & - & - & - & - & - & - & 0 & $0.0 \%$ \\
Meropenem & 6 & $4.1 \%$ & 25 & $25.8 \%$ & 17 & $17.0 \%$ & - & - \\
Moxifloxacin & - & - & - & - & - & - & 0 & $0.0 \%$ \\
Mupirocin & - & - & - & - & - & - & 0 & $0.0 \%$ \\
Nitrfurantoin & 9 & $6.1 \%$ & 57 & $58.8 \%$ & 97 & $98.0 \%$ & 1 & $1.5 \%$ \\
Oxacillin & - & - & - & - & - & - & 39 & $58.2 \%$ \\
Pencillin-g & 0 & $0.0 \%$ & - & - & - & - & 65 & $97.0 \%$ \\
Piperacillin-tazobactam & 95 & $64.2 \%$ & 53 & $54.1 \%$ & 21 & $21.0 \%$ & 0 & $0.0 \%$ \\
Rifampin & 1 & $100.0 \%$ & - & - & - & - & 2 & $3.1 \%$ \\
Tegecycline & 15 & $10.2 \%$ & 49 & $50.0 \%$ & 97 & $98.0 \%$ & - & - \\
Teicoplanin & - & - & - & - & - & - & 0 & $0.0 \%$ \\
Trime/sulphamethoxazole & 85 & $57.4 \%$ & 52 & $53.1 \%$ & 94 & $94.0 \%$ & 8 & $12.9 \%$ \\
Vancomycin & - & - & - & - & - & - & 0 & $0.0 \%$ \\
\hline \hline & & & & & & & &
\end{tabular}


TABLE VI: ASSOCIATION BETWEEN THE TYPE OF INFECTION AND STUDY VARIABLES

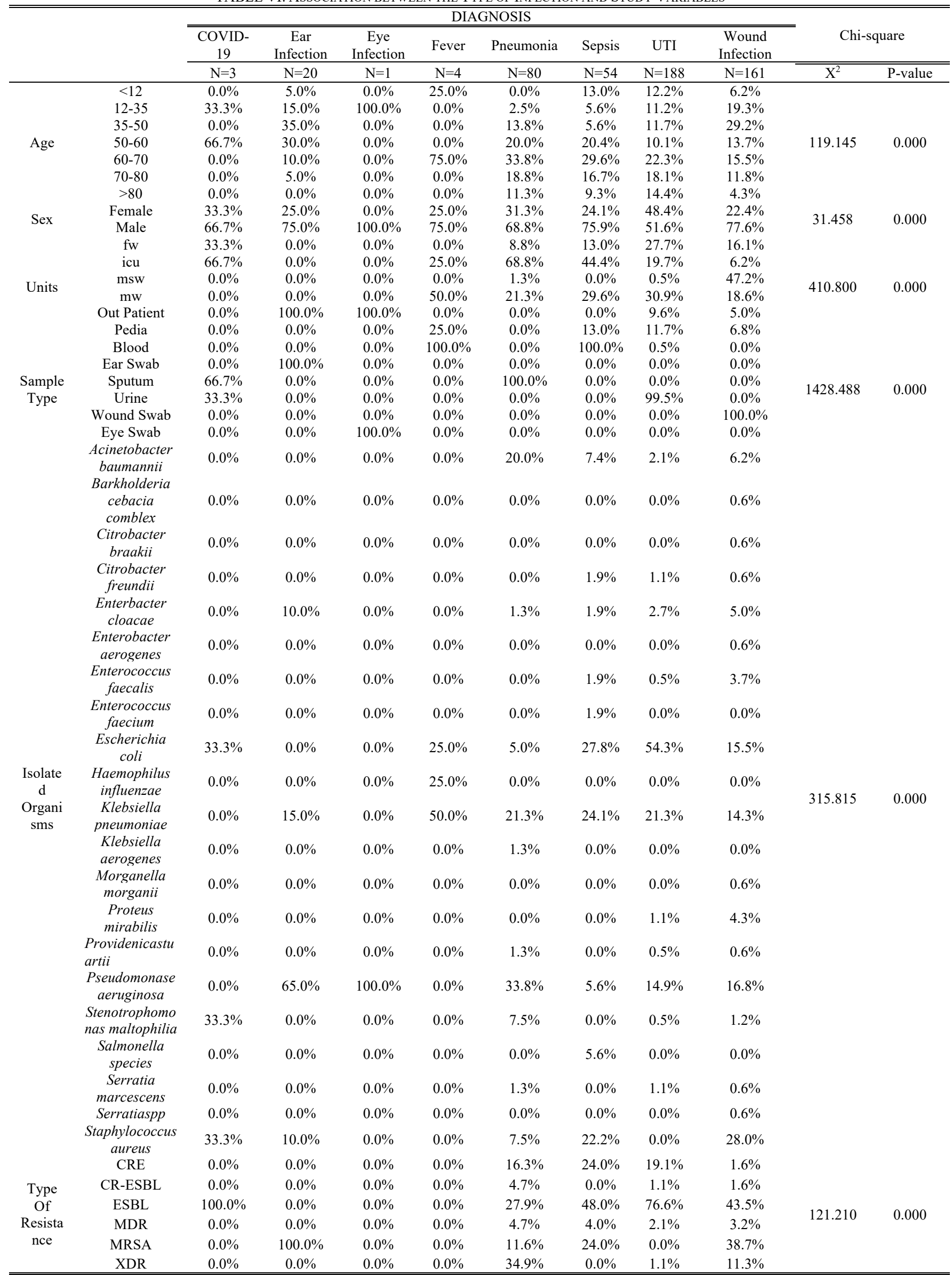




\section{E. Association Between the Type of Infection and Study Variables}

The adults (above 12 years and less than 60 years) and elder (above 60 years) ages were significantly associated with increased risk of infection including COVID-19, ear infection, eye infection, fever, pneumonia, sepsis, UTI and wound infection. Males showed high significant susceptibility to all diagnosed infections. COVID-19, pneumonia and sepsis showed a significant association with being admitted to ICU. Pseudomonas aeruginosa was significantly associated with pneumonia, ear, and eye infection. E. coli was significantly associated with most of the infections as COVID-19, fever, sepsis, UTI and wound infection. Klebsiella pneumoniae showed a significant association with pneumonia, sepsis and UTI. The type of resistance was significantly associated with the type of infection as ESBL was associated with COVID-19, pneumonia, sepsis, UTI and wound infection. MRSA was significantly associated with ear and wound infection (Table VI).

\section{DiscUSSION}

The world is striving to control COVID-19 pandemic, but on the other hand it is required to be equipped for facing the great influences of outbreak on AMR progression. There was evidence of an increase in the prevalence of multidrug resistant organisms (MDROs) specially during COVID-19 pandemic. Therefore, the impact of COVID-19 pandemic on AMR will only become clear in the coming years.

The present study showed that Escherichia coli, Pseudomonas aeruginosa, Staphylococcus aureus and Klebsiella pneumoniae were the most prevalent microorganisms isolated from the patients. Consistently, the most frequently isolated organisms were Klebsiella pneumoniae, Pseudomonas aeruginosa, extended-spectrum beta-lactamase, multidrug-resistant E. coli, Enterococcus, Chlamydia pneumoniae, Mycoplasma pneumoniae and Acinetobacter [9], [10].

Also, Pseudomonas aeruginosa was significantly associated with pneumonia, ear, and eye infection. E. coli was significantly associated with most of the infections as COVID-19, fever, sepsis, UTI and wound infection. Klebsiella pneumoniae showed a significant association with pneumonia, sepsis and UTI. In contrast, other studies showed that among COVID-19 patients, the bacteria most frequently isolated from were Mycoplasma pneumoniae, Pseudomonas aeruginosa, and Haemophilus influenzae. Over that coinfected patient with influenza showed different percentages of pathogenic isolates including Streptococcus pneumoniae and Streptococcus pyogenes as well as S. aureus [11].

The difference in incidence of resistance to antibiotics showed no significant change between 2019 and 2020. Several studies in Europe showed an increase in MDROs infections during COVID-19 pandemic but other studies in France and Spain did not show increase in MDROs infections. Therefore, the impact of COVID-19 pandemic on AMR will only become clear in the coming years as a lot of published data would become available [5]. It was noted that about $72 \%$ of COVID- 19 patients were treated with antibiotic even when not clinically indicated and this heavy use of empiric antibiotics led to high rate of AMR [6].

The usage of more antibiotics showed an increase in the ESBL which was associated with COVID-19, pneumonia, sepsis, UTI and wound infection. MRSA was significantly associated with ear and wound infection. ESBL and CRE were increased in 2019 than 2020. In the same respect, a study showed a wide spread of bacteria producing carbapenemase particularly among hospitalized patients. Also, the rate of carbapenem resistance involved 19.5\% MDR cultures and the rate of extended spectrum beta-lactamase was about $50 \%$ [12].

\section{CONCLUSION}

This study showed no significant difference in the AMR before and during the COVID-19 era. There still a debate around the implication of evolving AMR during COVID-19 outbreak thus other large population studies must be conducted to evaluate worldwide evolution of AMR during COVID-19 pandemic.

\section{REFERENCES}

[1] M. Dryden, A. P. Johnson, D. Ashiru-Oredope and M. Sharland, "Using antibiotics responsibly: right drug, right time, right dose, right duration," The Journal of Antimicrobial Chemotherapy 66(11):2441-3, 2011. doi:10.1093/jac/dkr370.

[2] T. M. Rawson, D. Ming, R. Ahmad, L. S. P. Moore and A. H. Holmes, "Antimicrobial use, drug-resistant infections and COVID-19," Nature Reviews Microbiology 18(8):409-10, 2020. doi:10.1038/s41579-0200395-y.

[3] T. M. Rawson, L. S. P. Moore, E. Castro-Sanchez, E. Charani, F. Davies, G. Satta and A. H. Holmes, "COVID-19 and the potential longterm impact on antimicrobial resistance," The Journal of Antimicrobial Chemotherapy. 75(7):1681-4, 2020. doi:10.1093/jac/dkaa194.

[4] M. A. B. Lucien, M. F. Canarie, P. E. Kilgore, G. Jean-Denis, N. Fénélon, M. Pierre, and B. Roman Pardo, "Antibiotics and antimicrobial resistance in the COVID-19 era: Perspective from resource-limited settings," International journal of infectious diseases 104:250-4, 2021. doi:10.1016/j.ijid.2020.12.087.

[5] D. L. Monnet and S. Harbarth "Will coronavirus disease (COVID-19) have an impact on antimicrobial resistance?" Euro Surveill 25(45):2001886, 2020. doi:10.2807/15607917.ES.2020.25.45.2001886.

[6] D. van Duin, G. Barlow and D. Nathwani, "The impact of the COVID19 pandemic on antimicrobial resistance: a debate," JAC Antimicrob Resist 2(3):dlaa053-dlaa, 2020. doi:10.1093/jacamr/dlaa053.

[7] R. Kohlmann and S. G. Gatermann, "Analysis and Presentation of Cumulative Antimicrobial Susceptibility Test Data--The Influence of Different Parameters in a Routine Clinical Microbiology Laboratory," PloS one 11(1):e0147965-e, 2016. doi:10.1371/journal.pone.0147965.

[8] CLSI.Performance standards for Antimicrobial Susceptibility Testing. $31^{\text {st }}$ ed. CLSI supplement M100. Clinical and laboratory standards Institute;2021.

[9] H. O. Ukuhor, "The interrelationships between antimicrobial resistance, COVID-19, past, and future pandemics," J Infect Public Health 14(1):53-60, 2021.

[10] L. Fattorini, R. Creti, C. Palma and A. Pantosti, "Bacterial coinfections in COVID-19: an underestimated adversary," 56:359-64, 2020. doi: 10.4415/ANN_20_03_14.

[11] L. Lansbury, B. Lim, V. Baskaran and W. S. Lim, "Co-infections in people with COVID-19: a systematic review and meta-analysis," Journal of Infection 81(2):266-75, 2020.

[12] B. Gasperini, A. Cherubini, M. Lucarelli, E. Espinosa and E. Prospero, "Multidrug-Resistant Bacterial Infections in Geriatric Hospitalized Patients before and after the COVID-19 Outbreak: Results from a Retrospective Observational Study in Two Geriatric Wards," Antibiotics 10(1), 95, 2021. doi:10.3390/antibiotics10010095. 